\title{
Report
}

\section{Comprehensive Treatment of Advanced Liver Cancer Complicated with Upper Gastrointestinal Bleeding in Multidisciplinary Team Model: A Case Report}

\author{
Liuming Lin, Mingrong Cao*, Qiang Li, Zhilong Liu \\ Department of Hepatobiliary Surgery, the First Affiliated Hospital of Jinan University, Guangzhou, China \\ Email address: \\ 547818974@qq.com (Liuming Lin), tcaomr@jnu.edu.cn (Mingrong Cao) \\ ${ }^{*}$ Corresponding author
}

\section{To cite this article:}

Liuming Lin, Mingrong Cao, Qiang Li, Zhilong Liu. Comprehensive Treatment of Advanced Liver Cancer Complicated with Upper Gastrointestinal Bleeding in Multidisciplinary Team Model: A Case Report. Journal of Surgery. Vol. 5, No. 3, 2017, pp. 47-52. doi: $10.11648 /$ j.js.20170503.13

Received: April 20, 2017; Accepted: May 4, 2017; Published: May 31, 2017

\begin{abstract}
A 44-year-old male was presented to emergency department because of repeated abdominal distension for 1 month, melena for 1 day and hematemesis for 4 hours. After examination, he was diagnosed with esophageal varices (III degree) and hemorrhage, primary massive hepatocellular carcinoma (cT3aN1Mx) and hepatitis B cirrhosis. In collaboration with the MDT team, this patient received a comprehensive treatment for up to 19 months which included surgical devascularization and cryoablation for liver cancer, TACE, SBRT, molecular targeted therapy, Chinese medicine treatment, antiviral therapy and so on. The latest review showed that liver cancer had distant metastasis, but the quality of life was still good. It seems that the comprehensive treatment of MDT is helpful for patients with HCC to improve the quality of life.
\end{abstract}

Keywords: Hepatocellular Carcinoma, Comprehensive Treatment, Multi-disciplinary Team

\section{Background}

Primary liver cancer is one of the main causes of cancer-related death worldwide. Cirrhosis is the most important risk factor for Hepatocellular Carcinoma (HCC). Hepatitis $\mathrm{B}$ and $\mathrm{C}$ are independent risk factors for liver cirrhosis. The pathogenesis of HCC is concealed and many of them were discovered with the initial diagnosis of liver cirrhosis with portal hypertension. There are a variety of treatment methods for liver cancer but only the orthotopic liver transplantation (OLT) or surgical resection can cure. There are also other treatments including arterial chemoembolization, radiofrequency ablation, microwave ablation, percutaneous ethanol injection, cryoablation, radiation therapy, systemic chemotherapy and molecular targeted therapy. Treatment options are based on tumor size, location, extrahepatic metastasis, and liver function [1].

Multidisciplinary team (MDT) is a clinical model proposed by American scholars in 1990s, in MDT, there is a fixed group of 2 or more medical experts from more than 2 disciplines. In view of the cases of a certain type or system, the experts put forward some suggestions from the aspects of diagnosis and treatment. The suggestions are analyzed comprehensively to draw up a suitable scheme for patients. The advantage of MDT is to transform the traditional model of individual experience into a scientific group decision-making system. For HCC, it is necessary to improve the long-term survival rate of patients by carrying out comprehensive treatment under MDT mode. For patients with advanced HCC, they are often affected by cancer pain, cachexia, indigestion and other symptoms. At the same time, excessive treatment may increase their financial burden and worsen their mood. These all result in a sharp decline in quality of life. So it is not the main purpose to completely cure the tumor, instead, we should concentrate on improving the quality of life. 


\section{Case Report}

The patient, a 44-year-old male, was presented to emergency department on September 8th, 2015 because of repeated abdominal distension for 1 month, melena for 1 day, hematemesis for 4 hours with a provisional diagnosis of acute upper gastrointestinal bleeding. Past medical history: 20 years of hepatitis B and chronic gastric ulcer for 8 years were not treated. Physical examination: blood pressure: $97 / 65 \mathrm{mmHg}$. Liver palms is visible. The liver enlarged with its lower edge $3 \mathrm{~cm}$ below the right costal margin and $8 \mathrm{~cm}$ below the xiphoid. Related examinations were consummated after hospitalization: Hemoglobin: $73.00 \mathrm{~g} / \mathrm{L}$, platelet count: $54.00 * 10^{\wedge} 9 / \mathrm{L}$, white blood cell count: $2.33 * 10^{\wedge} 9 / \mathrm{L}$, hepatitis B virus DNA: $5.26 \mathrm{E}+03 \mathrm{IU} / \mathrm{ml}$, alpha fetoprotein (AFP): $2584.7 \mathrm{ng} / \mathrm{ml}$. There was no active bleeding but esophageal varices (III degree) in the gastroscopy (Figure 1). The abdominal CT scan with contrast demonstrated a massive hepatocellular carcinoma of right hepatic lobe with intra-abdominal and retroperitoneal lymph node metastasis, portal hypertension, esophageal varices, and splenomegaly (Figure 2). The liver multidisciplinary team (MDT), including hepatobiliary surgeon, hepatologist, oncologist, radiologist, transplant physician/surgeon and traditional Chinese physician, held an emergency meeting to discuss the patient. According to the clinical manifestations and examinations, he was diagnosed with esophageal varices (III degree) and hemorrhage, primary massive hepatocellular carcinoma (cT3aN1Mx), hepatitis B cirrhosis. After a transient medical treatment, the reviewed hemoglobin was $71.2 \mathrm{~g} / \mathrm{L}$ on September 10th. Considering the risk of rebleeding, the MDT recommended that he should receive an

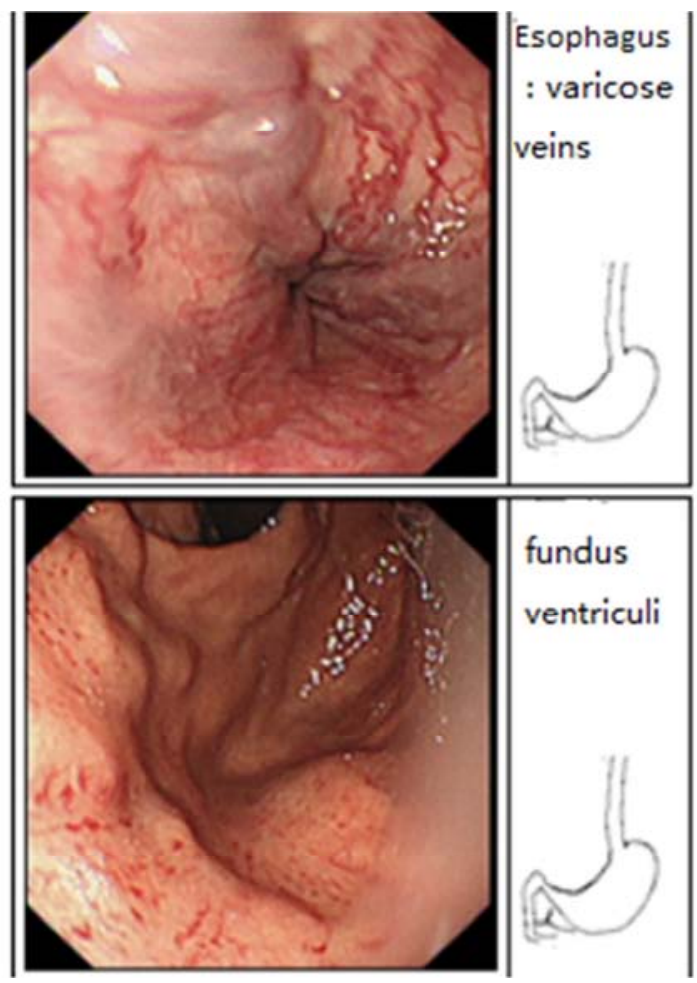

operation. Before that the hepatologist did a preoperative evaluation: the patient had no ascites and hepatic encephalopathy, total bilirubin and prothrombin time were normal, albumin was $32.9 \mathrm{~g} / \mathrm{L}$, suggesting the patient's liver function was Child class A. Emergency operation of pericardial devascularization, splenectomy and cryoablation for liver cancer was performed on September 10th, 2015. During operation severe liver cirrhosis and the giant tumor of right liver with a diameter of about $10 \mathrm{~cm}$ were observed which might lead to hepatic failure after resection because the compensatory hyperplasia of left liver was insufficient. So the cryoablation was performed instead. 8 straight probes were inserted into the tumor respectively and evenly distributed. After frozen for 15 minutes the probes were rewarming for 2 minutes. The tumor covered with ice can be observed. Then the process was repeated once again. Afterwards, probes were pulled out and inserted into the remaining tumors for cryoablation in the same way. The surgery went well and the hemoglobin continued to rise after the operation. On September 23rd, hemoglobin rose to $98 \mathrm{~g} / \mathrm{L}$ and AFP decreased to $1471.6 \mathrm{ng} / \mathrm{ml}$. The symptoms of abdominal distension, hematemesis, melena disappeared. Then the traditional Chinese physician and hepatologist recommended that the patient should take Ganfule tablet and Huaier granule as antineoplastic adjunctive therapy and entecavir as antiviral therapy continuously. The Ganfule tablet was given 6 tablets per time, three times per day in a treatment course for successive 2 months while the Huaier granule was given 1 bag per time, three times per day in a treatment course for successive 30 days. The patient was discharged on September 24, 2015.

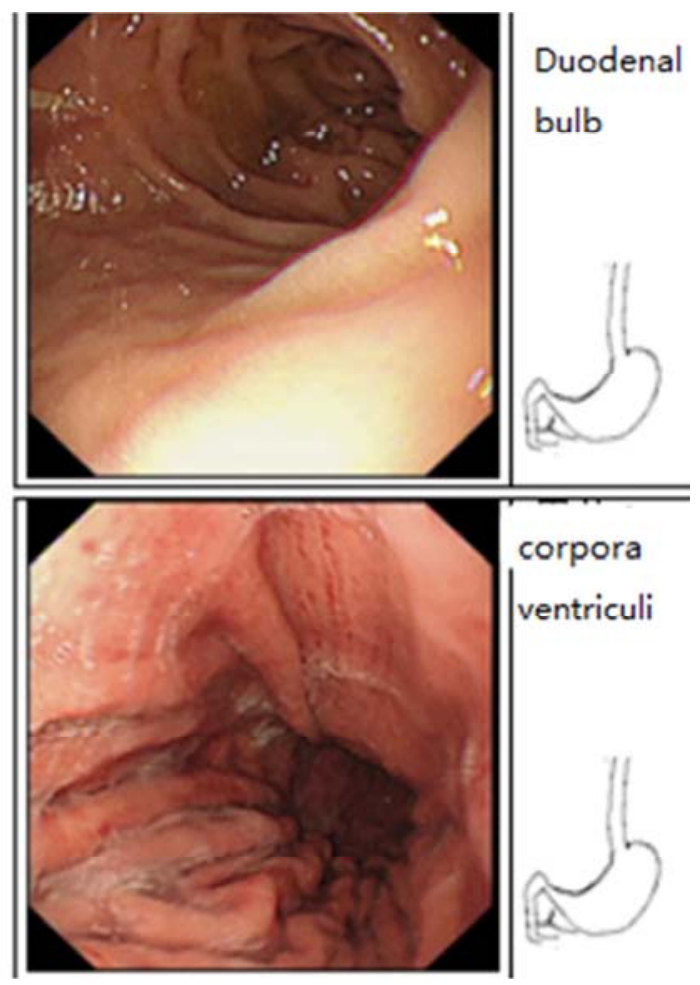

Figure 1. The varicose veins in the lower esophagus looked like nodules with a diameter of 0.6cm. Gastric fundus and body: The mucosa was congestive and scattered spotty erythema can be found. 

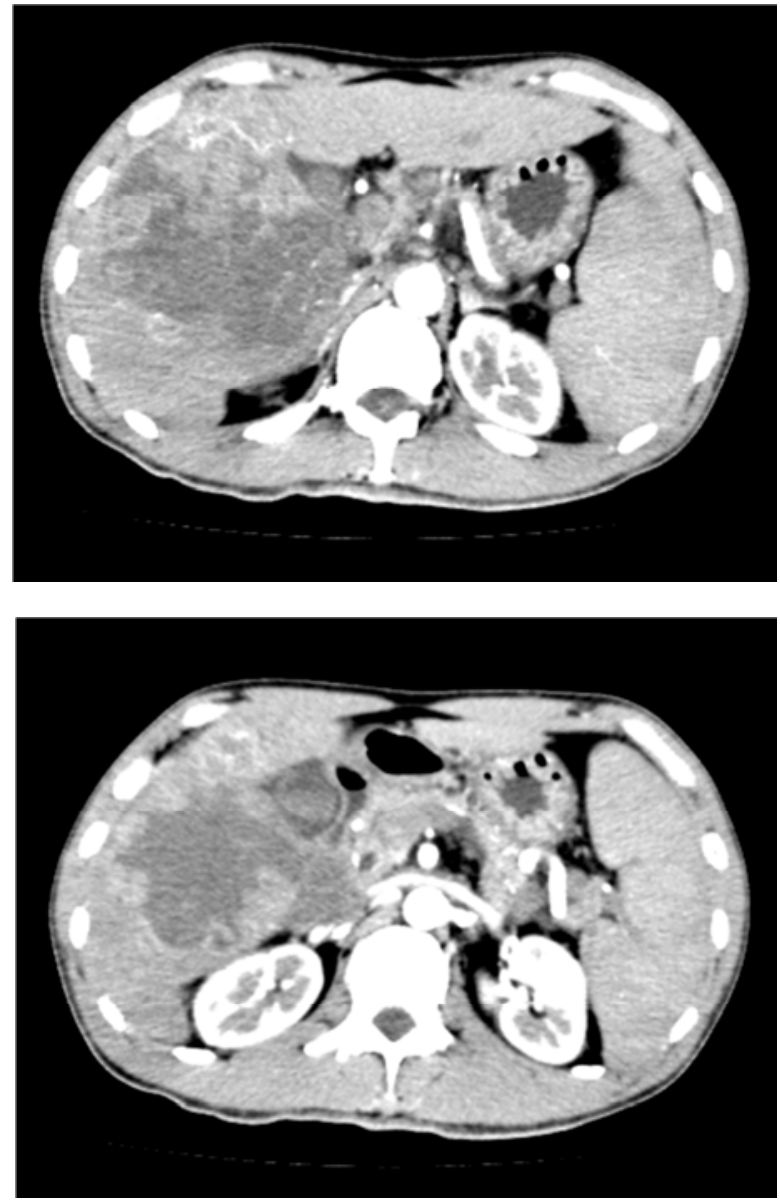

Figure 2. The abdominal CT scan with contrast demonstrated a massive hepatocellular carcinoma of right hepatic lobe with intra-abdominal and retroperitoneal lymph node metastasis, portal hypertension, esophageal varices, and splenomegaly. The size of the tumor was about $6 \mathrm{~cm} * 10 \mathrm{~cm} * 9 \mathrm{~cm}$.

On October 26th, 2015, the patient returned to the hospital to review and did not complain discomfort. Rechecked AFP was $1835.1 \mathrm{ng} / \mathrm{ml}$. Review of abdominal MR: the intralesional necrosis of right hepatocellular carcinoma increased than before and the front margin of pericardium was enhanced which might indicate the metastasis of the lymph node. The liver MDT held a meeting on November 2nd, 2015. The hepatologist and radiologist suggested to perform transcatheter artery chemotherapy embolization (TACE) to kill the remaining liver tumor. After discussion, the team recommended that he should receive the operation of TACE. After surgery, dizziness, vomiting and other side-effects were seen and then relieved by symptomatic treatment. The patient was discharged on November 6th, 2015.

The patient came back to review on January 25th, 2016. He did not complain of abdominal pain, distension or anorexia. AFP decreased to $1739.6 \mathrm{ng} / \mathrm{ml}$. Abdominal MR showed that the size of the right liver tumor decreased. At the meeting of MDT, the opinion of experts reached a basic agreement, recommending that he should receive the stereotactic body radiation therapy (SBRT) for liver cancer. During the period, the side-effect of myelosuppression appeared resulting in leukopenia. Then it was cured by symptomatic treatment. After repeated review, AFP gradually decreased. The abdominal MR on July 12th showed that the size of the right liver tumor and metastatic lymph nodes of peripancreas, para-aortic and front pericardium has further decreased than before (Figure 3). The PET/CT on July 19th showed that there were mixed density nodules in right hepatic lobe and caudate lobe. The area of cystic degeneration and iodine oil deposition demonstrated less glucose metabolism while it increased in other areas of tumor. Right axillary lymph node enlarged with increased glucose metabolism which was considered as metastatic tumor. The glucose metabolism of right parasternal and superior phrenic lymph nodes slightly increased which was considered as the possibility of metastasis. In the rest area of the body, there was no significant increase of fluorodeoxyglucose (FDG) metabolism. The liver MDT held a meeting again to discuss the next treatment. At the meeting, the oncologist suggested that distant metastasis is an indication of molecular targeted therapy. Due to the distant metastasis of $\mathrm{HCC}$, it was recommended that the patient take Sorafenib tablets as molecular targeting treatment. After four months of this treatment, however, the patient was unable to tolerate the adverse reactions, so he had to stopped taking the drugs.
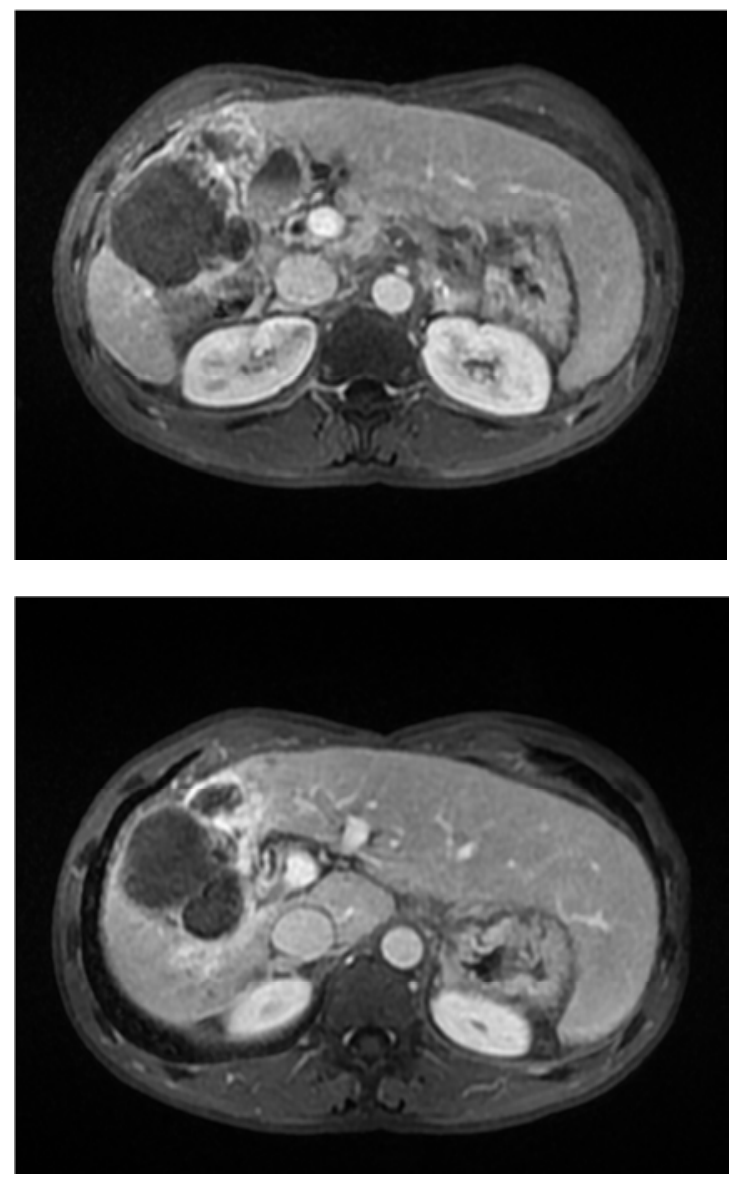

Figure 3. The abdominal MR showed that the size of the right liver tumor and metastatic lymph nodes of peripancreas, para-aortic and front pericardium decreased further than before. The size of the tumor was about $7 \mathrm{~cm} * 6 \mathrm{~cm} * 6 \mathrm{~cm}$. Compensatory hyperplasia of left lobe of liver was obvious. 
On November 11th, 2016, the patient returned to the hospital to review. Gastroscopy showed esophageal varices (III) and endoscopic variceal ligation was carried out. AFP reached a new low of $1024.6 \mathrm{ng} / \mathrm{ml}$. However, it bounced back to $3034.66 \mathrm{ng} / \mathrm{ml}$ which was much higher than ever in the review of February 13th, 2017. The next day, gastroscopic examination showed two varicose veins were found in the lower esophagus and they were ligated afterwards. The abdominal MR on February 16th suggested the size of tumor increased with the tumor embolus formation in left portal vein and that para-aortic metastatic lymph node enlarged. After the discussion of MDT, since the patient was in the terminal stage of hepatocellular carcinoma which kept progressing. Although it can not be cured, symptomatic treatments should be adopted to improve the quality of life. The patient was discharged from hospital without discomfort. Following up to April 2017, the total duration of treatment has reached 19 months and the patient is still under further treatment. Now we would like to share some of the experience in this case.

\section{Discussion}

$\mathrm{HCC}$ is closely related to liver cirrhosis. The hemorrhage from esophageal varices is one of serious complications and common causes of death in cirrhosis. It is not rare to discover HCC incidentally while patients are hospitalized for esophageal and gastric variceal bleeding (EGVB). The patient in this case was one of them. He received the emergency operation of pericardial devascularization, splenectomy and cryoablation for liver cancer. Devascularization has many advantages, such as bringing less damage to liver function, complete hemostasis effect, low difficulty and so on. Meanwhile, splenectomy can correct the imbalance of $\mathrm{Th} 1 / \mathrm{Th} 2$ which is common in patients with liver cirrhosis and correct hypersplenism, it is beneficial to the subsequent anti-tumor therapy. The patient's general condition and liver function were acceptable so that the treatment for HCC can be taken simultaneously. Such cases as resection of $\mathrm{HCC}$ with simultaneous splenectomy and devascularization does not increase mortality have been reported in china before [2]. Compared with HCC resection combined with devascularization, radiofrequency ablation combined with devascularization has less complications, brings less damage to liver function while long-term survival rate is similar [3]. Due to the lack of compensatory hyperplasia of the left liver, there was a risk of liver failure after hepatectomy, so the cryoablation was performed instead. In the NCCN 2016 guidelines for liver cancer, in the case of unresectable liver cancer, local therapy (ablation combined with arterial directed therapy) should be first considered when there is no condition for liver transplantation. It has been suggested that cryotherapy has become an important treatment of unresectable liver cancer [4]. After cryoablation, the levels of T cell subsets and NK cells in patients increased which improve the immune function and plays a role in anti-tumor effect [5]. After surgery, the patient's condition was stable and the AFP decreased obviously, which showed that the treatment had obvious inhibitory effect on HCC. During the treatment, the patient had kept taking Ganfule tablet and Huaier granule as adjuvant therapy. Ganfule tablet and Huaier granule are traditional Chinese medicine complex which are widely used for treatment of liver cancer in China. They are often used as adjuvant therapy. Traditional Chinese medicine treatment is one of the most important characteristics of anti-tumor therapy in China. In the past, this treatment was taken totally by the personal clinicians, the treating effect depended on their experiences but had no evidences to support. However, more and more evidences show that traditional Chinese medicine has its unique effect in anti-tumor therapy nowadays. In recent years, studies have shown that Ganfule tablet and Huaier granule may be beneficial for patients of HCC. In the prospective randomized controlled study on advanced primary hepatic cancer treated by ganfule prescription, it was concluded that the oral administration of Ganfule prescription could improve the quality of life of patients of primary hepatic cancer, decrease the serum AFP level and maintain the disease control rate and the one-year survival rate [6]. In the study on the clinical efficacy and safety of Huaier Granules combined with chemoembolization in treating primary liver cancer, 96 patients with liver cancer were randomly divided into the treatment group and the control group, 48 cases in each group. The control group used the chemoembolization therapy and the treatment group received additional Huaier Granules treatment. The result showed that Huaier Granules combined with chemoembolization in treating primary liver cancer could promote tumor necrosis, increase the patient's own immunity and improve the living quality with little adverse reactions, which is worth for promoting in clinical practice [7]. At the same time, the patient's HBV-DNA copies were significant high, he needs a long-term antiviral therapy (entecavir). Studies have shown that HBV may be reactivated during the treatment of liver cancer, thus result in liver dysfunction, which affects the subsequent treatment and leads to the final death [8]. The activity of HBV is also associated with recurrence [9]. The American Association for the Study of Liver Diseases (AASLD) and the Asian Pacific Association for the Study of Liver (APASL) have pointed out that HBV-associated hepatocellular carcinoma should be treated with antiviral therapy [10][11].

After the above treatments, the MR examination on October 2015 showed that the necrosis of right liver lesions increased, AFP increased slightly compared with the previous. In consideration of the good general condition and liver function of the patient, TACE combined with SBRT, one of the common treatments for unresectable hepatocellular carcinoma were recommended by the MDT. The latest NCCN guidelines suggested that TACE should be considered first for unresectable HCC with a diameter more than $5 \mathrm{~cm}$. More and more evidences indicated that SBRT is useful in liver cancer, and SBRT can be chosen as an alternative treatment when ablation or embolization was contraindicated or failed. For patients with a larger tumor diameter, SBRT can be used as a treatment option as long as liver function 
(Child-Pugh class A-B) and liver reserve capacity are good. MB Meng and colleagues made a meta-analysis to evaluate the efficacy and safety of transcatheter arterial chemoembolization (TACE) plus radiotherapy (RT) compared with TACE alone (Therapy I versus II) in treating unresectable hepatocellular carcinoma (UHCC). The analysis included 17 trials involving 1476 patients. 5 of total were Randomized Controlled Trials (RCTs) and 12 were Non-randomized Controlled Clinical Trials (CCTs). The result showed therapy I, compared with Therapy II, significantly improved the survival and the tumor response of patients, and was thus more therapeutically beneficial. Serious adverse events were not increased except total bilirubin (TB) level [12]. All of these show the application prospects of TACE combined with radiotherapy in advanced liver cancer treatment.

After the combined therapy, the AFP continued to decline and the patient was in good condition. However, PET/CT in July 2016 suggested that HCC had distant metastasis. In order to control the progression of the tumor, the patient was given a molecular targeted drug (Sorafenib). Sorafenib is a novel small molecule tyrosine kinase inhibitor which can inhibit the tyrosine receptor associated with tumor angiogenesis and development, thereby reduce the angiogenesis of tumor and inhibiting the growth of tumor. The NCCN guidelines suggested that molecular targeted therapy should be preferred for patients with distant metastases of liver cancer and Child-Pugh class A-B. But unfortunately, the patient can not tolerate the adverse reaction, which leads to the drug withdrawal. On February 2017, AFP reached 3034.66ng/ml, significantly higher than the previous. The abdominal MR showed that the right liver tumor and extrahepatic metastasis lymph nodes enlarged, suggesting that further progress of liver cancer. MDT suggested that the patient had been in the terminal stage of $\mathrm{HCC}$ and recommended symptomatic treatment to improve the quality of life.

The MDT mode played a very important role in the whole course of treatment, and had a profound impact on the diagnosis and treatment decisions. The liver MDT of our hospital consists of hepatobiliary surgeon, hepatologist, oncologist, radiologist, transplant physician/surgeon and traditional Chinese physician, and meets once every 2 weeks as a group to make consensus diagnostic and management recommendations for patients of liver diseases. By coordinating resources and minimizing communication failures, the MDT can quickly and efficiently route patients to appropriate therapeutic regimens and thereby optimize outcomes. According to the AASLD Practice Guidelines for the management of HCC, the complexity of HCC disease indicates that it should be managed in multidisciplinary setting [10]. In an evaluation of the early results of implementing a multidisciplinary management team for $\mathrm{HCC}$ at a Veterans Affairs Medical Center in San Francisco, the establishment of a MDT resulted in the doubling of patient referrals for treatment. Significantly more patients were evaluated at earlier stages of disease and received either palliative or curative therapies. The overall survival and length of follow-up were significantly improved after the establishment of the MDT [13]. In another research on evaluating differences in overall survival in patients with HCC after the establishment of a MDT for HCC, a total of 105 patients were diagnosed in the time period after HCC MDT initiation compared to 250 patients in the previous 4 years. The result showed that patients diagnosed after HCC MDT initiation had a median survival of 13.2 months compared to the 4.8 months observed in patients diagnosed before MDT formation [14]. These evidences suggested that the MDT model could benefit patients with HCC.

From this case we can see that the patients underwent surgical devascularization and cryoablation for liver cancer, TACE, SBRT, molecular targeted therapy, supplementing with Chinese medicine treatment, antiviral therapy and other adjuvant therapy. The liver MDT held a total of 6 meetings. At each stage of treatment, the suitable treatment options can be obtained through the MDT meeting. Meanwhile, avoiding unnecessary treatment reduced the financial burden of the patient. At present, he is still alive with cancer and has no symptoms of advanced HHC or cirrhosis such as abdominal pain or distension, anorexia, fatigue. From beginning to end, the patient's emotion is stable and there is no much financial burden. It has little effect on his daily life and work.

In conclusion, the treatment mode of MDT seems to be beneficial for patients with HCC, comprehensive treatment may improve the quality of life. It is worth promoting in the hospital where conditions are favorable, but more prospective studies are needed.

\section{References}

[1] Balogh J, Victor D, Asham E H, et al. Hepatocellular carcinoma: a review [J]. Journal of Hepatocellular Carcinoma, 2016, 3: 41-53.

[2] Shen Q, Xue H, Jiang Q. Surgical treatment of primary liver cancer complicated with portal hypertension [J]. Journal of Practical Diagnosis \& Therapy, 2007.

[3] Ding Z H, Mu Y, Zhang L, et al. Radiofrequency ablation combined with devascularization in treati ng primary liver cancer with portal hypertension: An analysis of 69 cases, 2013, 33 (11): 967-969.

[4] Xu K C, Niu L Z. Cryosurgery for hepatocellular carcinoma [J]. World Chinese Journal of Digestology, 2008, 16 (3): 229-235.

[5] Wang Z L, Zhang Y W. Progress on cellular immune function after the cryosurgical treatment of various cancers $[\mathrm{J}]$. Chinese Journal of Interventional Imaging \& Therapy, 2012, 9 (7): 556-559.

[6] Gao J L. [Prospective randomized controlled study on advanced primary hepatic cancer treated by ganfule prescription] [J]. China Journal of Chinese Materia Medica, 2014, 39 (12): 2367.

[7] Wu H, Hospital A N, University N M. Huaier Granules Combined with Chemoembolization in Treating Primary Liver Cancer in 96 Cases [J]. China Pharmaceuticals, 2014. 
[8] Jang J W. Hepatitis B virus reactivation in patients with hepatocellular carcinoma undergoing anti-cancer therapy $[\mathrm{J}]$. World Journal of Gastroenterology Wjg, 2014, 20 (24): 7675.

[9] Ishikawa T. Anti-viral therapy to reduce recurrence and improve survival in hepatitis B virus-related hepatocellular carcinoma [J]. World Journal of Gastroenterology, 2013, 19 (47): 8861-8866.

[10] Bruix J, Sherman M. Management of hepatocellular carcinoma: an update. american association for the study of liver diseases (AASLD) practice guideline [J]. 2011, 53: 1020-1035.

[11] Omata M, Lesmana L A, Tateishi R, et al. Asian Pacific Association for the Study of the Liver consensus recommendations on hepatocellular carcinoma $[\mathrm{J}]$. Hepatology International, 2010, 4 (2): 439.
[12] Meng M B, Cui Y L, Lu Y, et al. Transcatheter arterial chemoembolization in combination with radiotherapy for unresectable hepatocellular carcinoma: a systematic review and meta-analysis. [J]. Radiotherapy \& Oncology, 2009, 92 (2): 184-194.

[13] Chang T T, Sawhney R, Monto A, et al. Implementation of a multidisciplinary treatment team for hepatocellular cancer at a Veterans Affairs Medical Center improves survival [J]. HPB, 2008, 10 (6): 405-411.

[14] Yopp A C, Mansour J C, Beg M S. Establishment of a Multidisciplinary Hepatocellular Carcinoma Clinic is Associated with Improved Clinical Outcome [J]. Annals of Surgical Oncology, 2014, 21 (4): 1287-95. 